\title{
Soliton dynamics in finite nonlocal media with cylindrical symmetry
}

\author{
Emmanuel Garza, ${ }^{1}$ Servando Lopez-Aguayo, ${ }^{2,}{ }^{*}$ and Julio C. Gutiérrez-Vega ${ }^{2}$ \\ ${ }^{1}$ Computing and Mathematical Sciences, California Institute of Technology, Pasadena, California 91125, USA \\ ${ }^{2}$ Photonics and Mathematical Optics Group, Tecnologico de Monterrey, Monterrey 64849, Mexico
}

(Received 28 August 2018; published 1 March 2019)

\begin{abstract}
The effect of finite boundaries in the propagation of spatial nonlocal solitons in media with cylindrical symmetry is analyzed. Using Ehrenfest's theorem together with the Green's function of the nonlinear refractive index equation, we derive an analytical expression for the force exerted on the soliton by the boundaries, verifying its validity by full numerical propagation. We show that the dynamics of the soliton are determined not only by the degree of nonlocality, but also by the boundary conditions for the refractive index. In particular, we report that a supercritical pitchfork bifurcation appears when the boundary condition exceed a certain threshold value.
\end{abstract}

DOI: 10.1103/PhysRevA.99.033804

\section{INTRODUCTION}

Nonlinear self-trapped optical beams, also known as spatial optical solitons, have been extensively studied over the years in several media for their potential use in all-optical communication networks $[1,2]$. Of special interest is the study of nonlocal solitons, which are present when the nonlinear response at a particular point of the medium not only depends on the optical field localized at that single point, but is a function of the intensity around the neighboring region. Examples of media with nonlocal processes are the diffusion of charge carriers [3], thermal media [4], and liquid crystals [5]. Nonlocality allows several phenomena that are not possible to observe in pure Kerr local media. For example, nonlocality can form new bound states and stable families of solitons such as Hermite solitons [6], Laguerre solitons [7], azimuthons [8], and ellipticons [9], among other self-trapped nonlinear optical beams. Additionally, the presence of finite boundaries in nonlocal media can induce transformations between solitons of different symmetries and exert repulsive forces [10,11]. In fact, there is the possibility that far-away asymmetric boundary forces can exert control over the soliton dynamics as it was shown in [12] for the case of propagation in highly nonlocal nonlinear media. Similarly, a power-dependent nonlinear repulsion at the boundary was reported in [13] and for the case of thermal optical nonlinearity, the range of action of the boundary is virtually infinite [14]. When this range of action is much larger than the width of the soliton, an equivalent particle theory can be used to describe the soliton dynamics [15].

In [16], Brambila and Fratalocchi studied the transport of energy in multidimensional quantum chaos, for which a general two-dimensional (2D) nonlinear quantum kicked rotor (which encompasses the 2D nonlinear Schrödinger equation) is the governing model. The approach is quite general and makes use of conserved quantities and dynamics of variational coordinates to find fully analytic solutions, concluding,

\footnotetext{
*servando@itesm.mx
}

remarkably, that solitons can facilitate the energy transport in a disordered medium. On the other hand, applications of the nonlocal Schrödinger equation have been studied for the controlled soliton splitting using a localized inhomogeneity [17], while [18] demonstrates that Landau-Zener tunneling can occur in one-dimensional lattices of undoped liquid-crystalline waveguides.

In particular, using the method of images and assuming that the soliton waist is much smaller than the radius of the boundary, Shou et al. [19] obtained an analytical approximation for the boundary force exerted on a soliton for the case of thermal self-focusing nonlinearity in lead glass with circular boundaries, which corresponds to the highly nonlocal limit. In this paper we extend the analysis of the circular cylinder to include the case of an arbitrary degree of nonlocality, as well as general Dirichlet boundary conditions for the nonlinear nonlocal refractive index. We report an analytical expression for the boundary force that shows good agreement with numerical results, revealing that the soliton dynamics for a general degree of nonlocality are significantly different from the purely nonlocal limit case.

\section{PHYSICAL MODEL}

We start our analysis by considering the nonlinear propagation of a monochromatic complex field envelope $U(X, Y, Z)$ in a nonlocal medium described by the nonlinear Schrödinger equation

$$
2 i k \frac{\partial U}{\partial Z}+\left(\frac{\partial^{2}}{\partial X^{2}}+\frac{\partial^{2}}{\partial Y^{2}}\right) U+2 k^{2} \frac{\Delta n\left(|U|^{2}\right)}{n_{0}} U=0
$$

where $Z$ is the propagation coordinate, $X$ and $Y$ are the transverse coordinates, $n_{0}$ is the linear refractive index of the medium, $k=\omega n_{0} / c$ is the wave number, and $\Delta n$ represents the nonlinear change in the refractive index that depends upon the optical field intensity distribution. Here we study the case where the nonlocal nonlinear response of the medium obeys 
the 2D screened Poisson equation [20]

$$
b\left(\frac{\partial^{2}}{\partial X^{2}}+\frac{\partial^{2}}{\partial Y^{2}}\right) \Delta n-a \Delta n+|U|^{2}=0,
$$

where $a$ and $b$ are parameters that depend on the particular properties of the medium. The model represented by (2) has been previously used to describe partially ionized plasmas [21], plasma heating on the propagation of electromagnetic waves [3], and a thermal nonlinear process in the regime of strong absorption [22]. In particular, a physical counterpart of the model given by (2) is used to study nematic liquid crystals in a planar cell that accounts for the observability of accessible solitons $[20,23,24]$. The ratio $a / b$ can be related to the degree of nonlocality; the case $a / b \rightarrow 0$ corresponds to highly nonlocal media, while $a / b \rightarrow \infty$ models pure Kerr media. We restrict ourselves to the case in which the medium has a finite cross section given by a circular boundary of radius $R_{b}$. Without loss of generality, we rescale the spatial coordinates by the relations $x=X / R_{b}, y=Y / R_{b}$, and $z=$ $Z / k R_{b}^{2}$ and introduce the normalized versions of the nonlinear response, field envelope, and nonlocal parameter given by $N=k^{2} R_{b}^{2} \Delta n / n_{0}, \Psi=k R_{b}^{2} U / \sqrt{b n_{0}}$, and $\rho=\sqrt{a / b} R_{b}$, respectively. Additionally, given the circular symmetry of the boundaries, we use cylindrical coordinates $(r, \theta, z)$ with $r=$ $\sqrt{x^{2}+y^{2}}, \theta=\arctan (y / x)$, and the position vector at a fixed $z$ defined by $\mathbf{r}=x \hat{i}+y \hat{j}$. Under these assumptions, the governing equations for the beam propagation (1) and (2) are now given by

$$
\begin{aligned}
i \frac{\partial \Psi}{\partial z}+\frac{1}{2} \nabla_{\perp}^{2} \Psi+N\left(|\Psi|^{2}\right) \Psi & =0, \quad r \leqslant 1, \quad z>0 \\
\Psi(\mathbf{r}, z) & =\Psi_{0}(\mathbf{r}), \quad z=0, \\
\rho^{2} N-\nabla_{\perp}^{2} N & =|\Psi|^{2}, \quad r<1, \quad z \geqslant 0 \\
N(\mathbf{r}, z) & =N_{b}(\theta), \quad r=1, z \geqslant 0,
\end{aligned}
$$

where $\nabla_{\perp}^{2}=\partial^{2} / \partial x^{2}+\partial^{2} / \partial y^{2}$ stands for the transverse Laplacian, and $N_{b}(\theta)$ is the prescribed Dirichlet boundary condition for the refractive index.

\section{RESULTS}

\section{A. Analytical results}

We continue our analysis by finding fundamental solitons centered at the origin by using the ansatz $\tilde{\Psi}(\mathbf{r}, z)=e^{i \lambda z} \psi(\mathbf{r})$, where $\lambda$ is the soliton's propagation constant and $\psi(\mathbf{r})$ is a real function. Thus, (3a) becomes

$$
\frac{1}{2} \nabla_{\perp}^{2} \psi+N\left(|\psi|^{2}\right) \psi-\lambda \psi=0,
$$

while in (3b) $\Psi$ is just replaced by $\psi$. Then, different soliton trajectories can be analyzed by adding a tilt to $\tilde{\Psi}$. The position of the soliton at a fixed propagation distance $z$ is characterized by the intensity centroid $\mathbf{r}_{c}=x_{c} \hat{i}+y_{c} \hat{j}$ using

$$
\mathbf{r}_{c}(z)=\frac{1}{P} \iint|\Psi(\mathbf{r}, z)|^{2} \mathbf{r} d x d y
$$

where the power of the soliton is

$$
P=\iint|\Psi|^{2} d x d y
$$

By introducing a finite medium where the refractive index is a solution to (3b), the launched soliton experiences a force whenever the beam center is not at the origin due to the asymmetry in the refractive index $N$ generated in order to meet the boundary conditions. The force exerted on the beam centroid by the boundaries can be described by Ehrenfest's theorem [20]

$$
\frac{d^{2} \mathbf{r}_{c}}{d z^{2}}=\frac{1}{P} \iint|\Psi(\mathbf{r}, z)|^{2} \nabla N(\mathbf{r}, z) d x d y .
$$

For the highly nonlocal limit, assuming that the soliton waist is much smaller than the radius of the waveguide, an analytical approximation to the boundary force was obtained in [19] using the method of images. This approximation is based on the assumption that the entire power of the beam is localized at the intensity centroid, i.e., $|\Psi(\mathbf{r}, z)|^{2} \approx P \delta\left(\mathbf{r}-\mathbf{r}_{c}(z)\right)$, where $\delta\left(\mathbf{r}-\mathbf{r}_{c}\right)$ is the Dirac delta function. Under this assumption, the induced refractive index $N$ is then given by the Green's function of (3b) (for a fixed $z$ ). In the case $\rho=0$ (Poisson's equation), the method of images can be used to find the Green's function, but for arbitrary $\rho$ we need to consider a different procedure. Assuming that the soliton can be modeled as $|\Psi|^{2}=P \delta\left(\mathbf{r}-\mathbf{r}_{c}\right)$, the induced refractive index can be found by solving the boundary-value problem

$$
\begin{aligned}
\rho^{2} G\left(\mathbf{r}, \mathbf{r}_{c}\right)-\nabla_{\perp}^{2} G\left(\mathbf{r}, \mathbf{r}_{c}\right) & =P \delta\left(\mathbf{r}-\mathbf{r}_{c}\right) \quad \text { for } r<1, \\
G\left(\mathbf{r}, \mathbf{r}_{c}\right) & =N_{b}(\theta) \quad \text { for } r=1,
\end{aligned}
$$

which we solve by using the method of separation of variables along with standard techniques for finding Green's functions [25,26]. We proceed to split $G$ into three components $G\left(\mathbf{r}, \mathbf{r}_{c}\right)=G_{1}\left(\mathbf{r}, \mathbf{r}_{c}\right)+G_{2}\left(\mathbf{r}, \mathbf{r}_{c}\right)+G_{H}(\mathbf{r})$, where $G_{1}\left(\mathbf{r}, \mathbf{r}_{c}\right)$ is the Green's function of the screened Poisson equation subject to the solution being finite at infinity, $G_{2}\left(\mathbf{r}, \mathbf{r}_{c}\right)$ is such that $G_{1}\left(\mathbf{r}, \mathbf{r}_{c}\right)+G_{2}\left(\mathbf{r}, \mathbf{r}_{c}\right)$ satisfies (8) with zero boundary conditions, and $G_{H}(\mathbf{r})$ is a homogeneous solution that satisfies the boundary condition. Each term is given explicitly by

$$
\begin{aligned}
G_{1}\left(\mathbf{r}, \mathbf{r}_{c}\right) & =\frac{P}{2 \pi} K_{0}\left(\left|\mathbf{r}-\mathbf{r}_{c}\right|\right) \\
G_{2}\left(\mathbf{r}, \mathbf{r}_{c}\right) & =-\frac{P}{\pi \rho r_{c}} \sum_{n=-\infty}^{\infty} \frac{K_{n}(\rho) I_{n}(\rho r) I_{n}\left(\rho r_{c}\right)}{I_{n}(\rho) F_{n}\left(\rho r_{c}\right)} \cos \left[n\left(\theta-\theta_{c}\right)\right] \\
G_{H}(\mathbf{r}) & =\sum_{n=-\infty}^{\infty} A_{n} \frac{I_{n}(\rho r)}{I_{n}(\rho)} e^{i n \theta},
\end{aligned}
$$

where $I_{n}$ and $K_{n}$ stand for the modified Bessel functions of the first and second kind, respectively, $A_{n}$ are the Fourier coefficients of $N_{b}(\theta)$, that is, $N_{b}(\theta)=\sum A_{n} e^{i n \theta}$, and

$$
\begin{aligned}
F_{n}\left(\rho r_{c}\right)= & I_{n}\left(\rho r_{c}\right)\left[K_{n+1}\left(\rho r_{c}\right)+K_{n-1}\left(\rho r_{c}\right)\right] \\
& +K_{n}\left(\rho r_{c}\right)\left[I_{n+1}\left(\rho r_{c}\right)+I_{n-1}\left(\rho r_{c}\right)\right] .
\end{aligned}
$$

The term $G_{1}$ is symmetric with respect to the beam center and thus provides the self-focusing effect that sustains the soliton [19]. On the other hand, the terms $G_{2}$ and $G_{H}$ produce a steering force on the beam. Using (7) and the approximation 
$|\Psi|^{2}=P \delta\left(\mathbf{r}-\mathbf{r}_{c}\right)$, we find that

$$
\begin{aligned}
\frac{d^{2} \mathbf{r}_{c}}{d z^{2}}= & \mathbf{F}_{2}\left(r_{c}\right)+\mathbf{F}_{H}\left(r_{c}, \theta_{c}\right), \\
\mathbf{F}_{2}\left(r_{c}\right)= & -\frac{P}{2 \pi r_{c}} \\
& \times \sum_{n=-\infty}^{\infty} \frac{K_{n}(\rho) I_{n}\left(\rho r_{c}\right)\left[I_{n+1}\left(\rho r_{c}\right)+I_{n-1}\left(\rho r_{c}\right)\right]}{I_{n}(\rho) F_{n}\left(\rho r_{c}\right)} \hat{\mathbf{r}},
\end{aligned}
$$

$$
\begin{aligned}
\mathbf{F}_{H}\left(r_{c}, \theta_{c}\right)= & \sum_{n=-\infty}^{\infty} \frac{A_{n}}{I_{n}(\rho)} e^{i n \theta_{c}}\left\{\frac{\rho}{2}\left[I_{n+1}\left(\rho r_{c}\right) I_{n-1}\left(\rho r_{c}\right)\right] \hat{\mathbf{r}}\right. \\
& \left.+i \frac{n}{r_{c}} I_{n}\left(\rho r_{c}\right) \hat{\boldsymbol{\theta}}\right\} .
\end{aligned}
$$

These equations constitute the main analytical result in this paper and describe the effect of finite boundaries on the propagation of spatial nonlocal solitons inside a circular cylinder.

\section{B. Numerical validation}

Next we validate the quality of the analytical approximation by means of direct numerical experimentation. In order to obtain fundamental soliton solutions, we use a nonlinear least-squares method applied to (4) and find the optimal parameters of a centered Gaussian beam of a given waist $\omega_{0}$ for unknown amplitude $A$ and propagation constant $\lambda$. This way we can control the width of the beam to satisfy $\omega_{0} \ll 1$, which guarantees the validity of (11). We find, by means of numerical experimentation, that using the restriction $\omega_{0}<0.2$, the soliton dynamics are described satisfactorily by our analytical results for at least 20 diffraction lengths. The solution for the refractive index in (3b) (for a fixed $z$ ) is found using a method similar to that of [27], in which a fast Fourier transform and a finite-difference scheme are used to discretize the polar and radial coordinates, respectively. We propagate the solitons by means of the split-step method [28].

In Fig. 1 we present several soliton trajectories predicted by the analytical model (right column) as well as the corresponding full numerical propagation (left column). First, we demonstrate that the centroid of the soliton remains fixed as long as we launch it at an equilibrium point, as it is shown in Fig. 1(a). On the other hand, Fig. 1(b) shows a circular orbit obtained by launching the soliton with an initial transverse momentum (tilt) balanced with the centripetal force generated by the boundary condition. The particular launching conditions for this case are $r_{c}(z=0)=0.5$ and $\Psi(\mathbf{r}, 0)=\Psi_{0}(\mathbf{r}-$ $\left.\mathbf{r}_{c}(0), 0\right) \exp \left(i g_{x} x+i g_{y} y\right)$, where we set $g_{x}=\sqrt{F_{r} r_{c}}=5.89$ and $g_{y}=0$. Similar soliton interactions in a nonlocal nonlinear medium analogous to gravitational forces were reported recently in [29]. In general, the soliton trajectories can be quite complex, as it is shown in Fig. 1(c), where a soliton is launched with $g_{x}=g_{y}=5.89$. Since the soliton experiences an acceleration, it radiates energy, thus decreasing the effective power of the soliton and changing the long-term dynamics [30]. Note that disregarding the long-term effects of wave radiation that are naturally generated due to the acceleration of
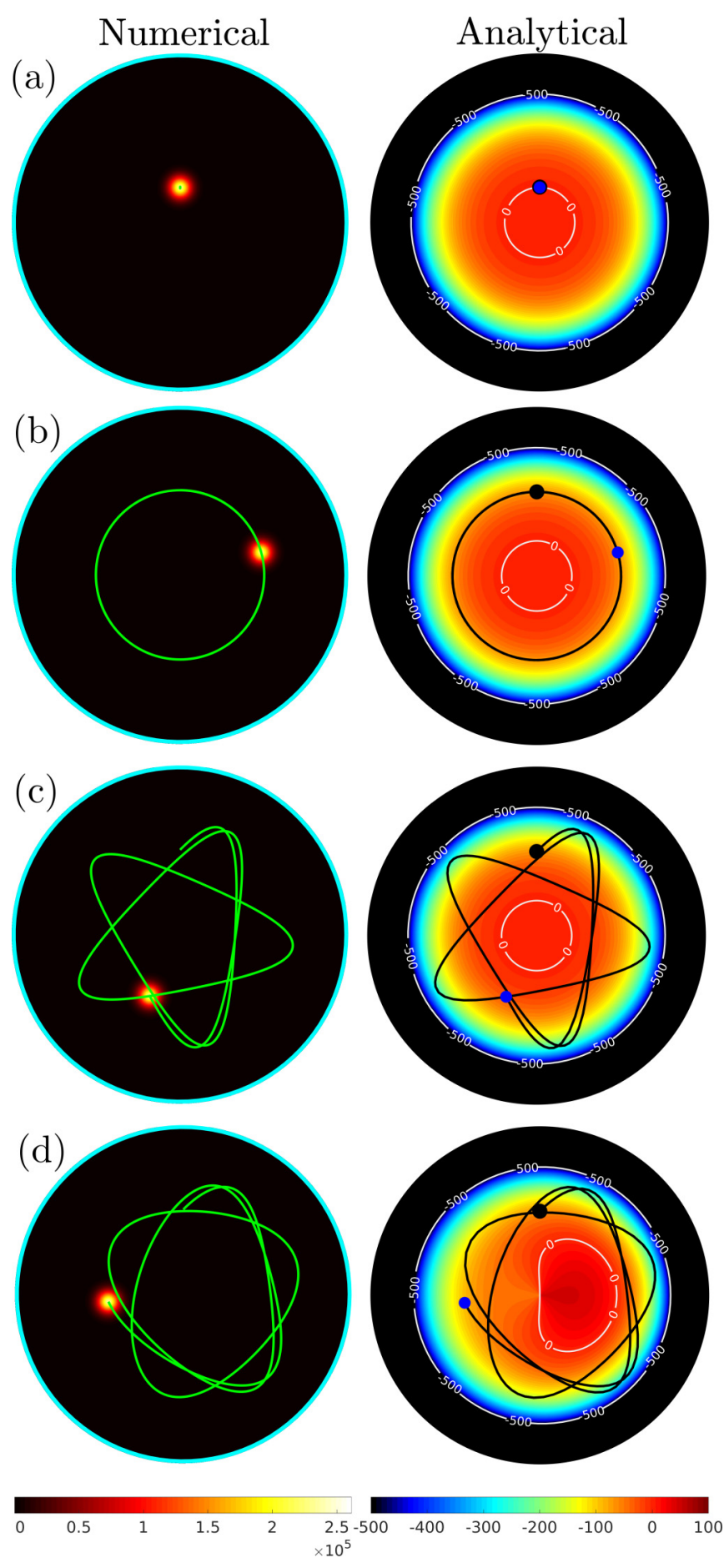

FIG. 1. Soliton trajectories for a variety of launching conditions. The left column corresponds to the path given by the full numerical propagation (shown in green) and the colormap represents the beam intensity $|\Psi|^{2}$ at $z=400 \pi / \lambda$. The right column presents the prediction by the analytical model (11), where the black curve is the predicted path of the soliton starting from the black marker and finishing on the blue one, and the colormap represents the radial force with contour lines of 0 and 500 also shown. For all cases $\rho=1, z \in$ $[0,400 \pi / \lambda]$, and $P=3000$. For (a)-(c) $N_{b}=900$ and in particular (a) shows a static soliton centered at an equilibrium point $y_{c}=0.21$, (b) presents a circular orbit of radius 0.5 , (c) has a trajectory bounded by a minimum and maximum radii, and (d) has an added angular modulation at the boundary given by $N_{b}(\theta)=900+50 \cos (\theta)$. 


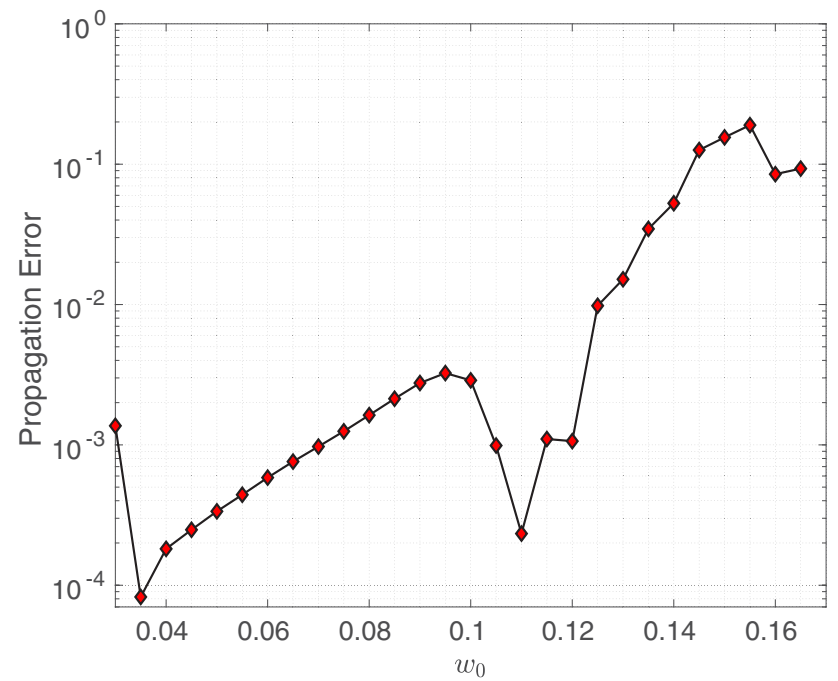

FIG. 2. Error in the soliton propagation as a function of the soliton's width. The error is measured as the distance between the position at a final time of the soliton propagated by the analytical model and the point of the soliton's centroid at that same time given by the numerical propagation. Here $\rho=1$ and the boundary conditions in Fig 1(d) were used. Note that for $\omega_{0} \leqslant 0.12$ we get errors of less than $10^{-2}$.

the beam [31] or the soliton breathing induced by the corresponding approximation used, the predicted analytical results are corroborated by full numerical propagation. Finally, we set the boundary condition to be $N_{b}=900+50 \cos (\theta)$ with the same initial conditions as in Fig. 1(c); the corresponding dynamics are shown in Fig. 1(d). In this case, note that the equilibrium region is no longer centered around $r=0$, nor it is of circular shape.

In order to validate the predicted trajectories as a function of the soliton waist $\omega_{0}$ in a quantitative way, Fig. 2 shows the error between the predicted final position of the soliton and the one obtained through the full numerical propagation. For the particular case of $\rho=1$, the approximation is better than $10^{-2}$ even for a beam waist of 0.12 (12\% of the radius of the boundaries). Additionally, Table I presents the propagation

TABLE I. Propagation error for values of $\omega_{0}$ equal to 0.08 and 0.15 and for different degrees of nonlocality $\rho$. We can see that the analytical approximation works best for the smaller soliton width and in general for higher values of $\rho$ (local effects are dominant) the approximation also worsens. The solitons launched for this test all had zero tilt and were displaced to the coordinate $(0.5,0)$ and propagated for a distance $100 \pi / \lambda$.

\begin{tabular}{lllc}
\hline \hline$\omega_{0}$ & $\rho$ & $P$ & Propagation error \\
\hline 0.08 & 0.1 & 3668 & $1.63 \times 10^{-4}$ \\
0.08 & 1 & 3695 & $1.71 \times 10^{-3}$ \\
0.08 & 5 & 4079 & $1.75 \times 10^{-2}$ \\
0.08 & 10 & 4841 & $9.64 \times 10^{-3}$ \\
0.15 & 0.1 & 1044 & $2.71 \times 10^{-3}$ \\
0.15 & 1 & 1065 & $2.48 \times 10^{-2}$ \\
0.15 & 5 & 1346 & $1.41 \times 10^{-1}$ \\
0.15 & 10 & 2089 & $6.41 \times 10^{-1}$ \\
\hline \hline
\end{tabular}
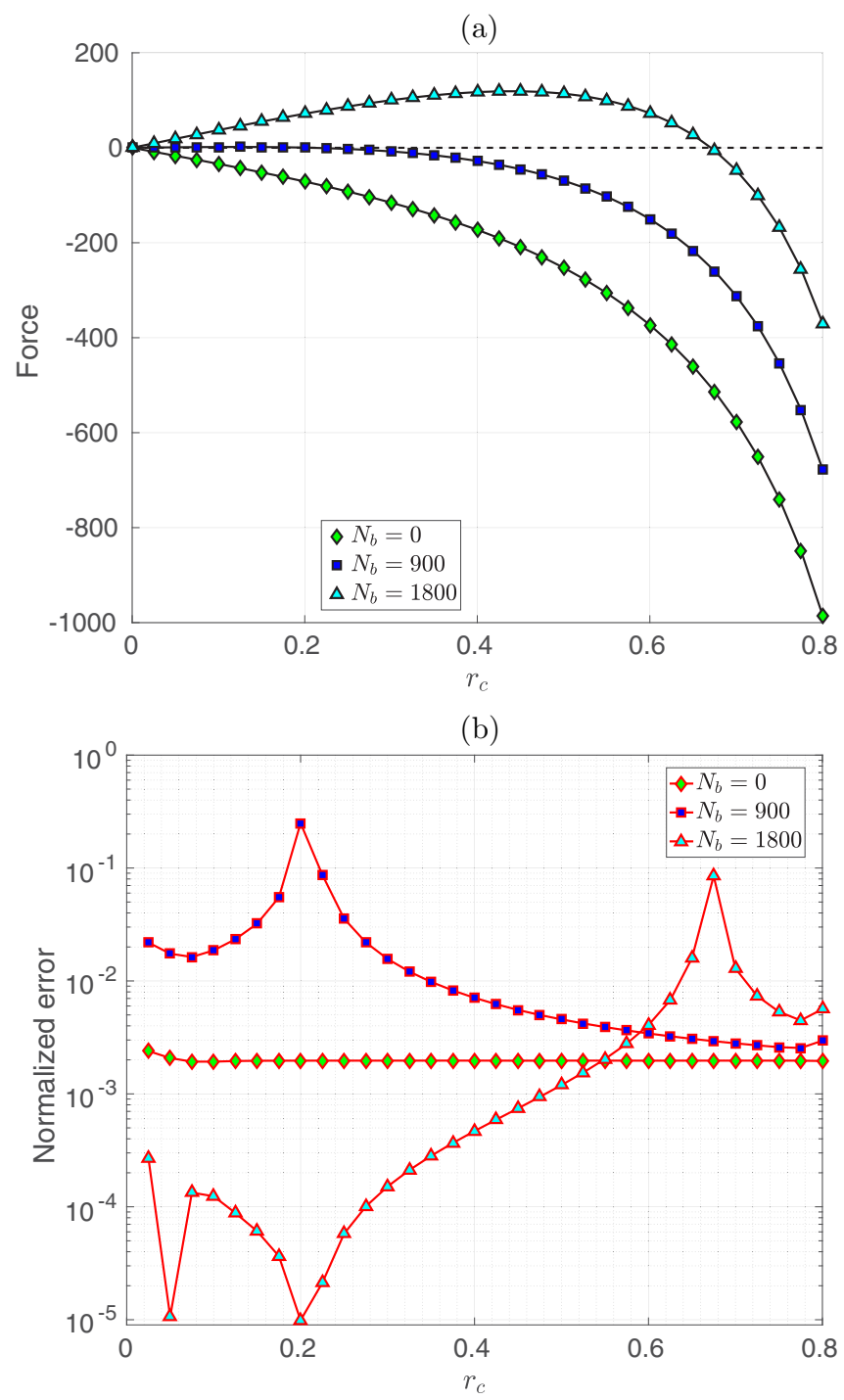

FIG. 3. Comparison of the numerically computed force using (7) and the analytical approximation (11) for a soliton with $P=3000$ and different values of $N_{b}$. In (a) the markers represent the values obtained by the numerical simulations, the black lines are the predicted values by the analytical model, and the dashed black line is the force $=0$ axis. In (b) we present the normalized errors between the numerical and analytical forces, showing good agreement between simulation and the analytical prediction. Note that the peaks at around $r_{c} \approx 0.2$ and $r_{c} \approx 0.7$ are caused by the normalization value which is close to zero.

errors for solitons with widths of 0.08 and 0.15 for various degrees of nonlocality. The results show that the approximation is fairly good for the smaller width beam and higher degrees of nonlocality (smaller $\rho$ ), while it fails to provide a meaningful predictions for larger widths and lower degrees of nonlocality. For example, using $\omega=0.15$ and $\rho=10$ generates a large error in the predicted final position and thus another approach should be considered.

A clear advantage of propagating using (11) over a full numerical propagation method is that with the analytical expressions, the computational time can be reduced by two or even three orders of magnitude, without losing significant 
(a)

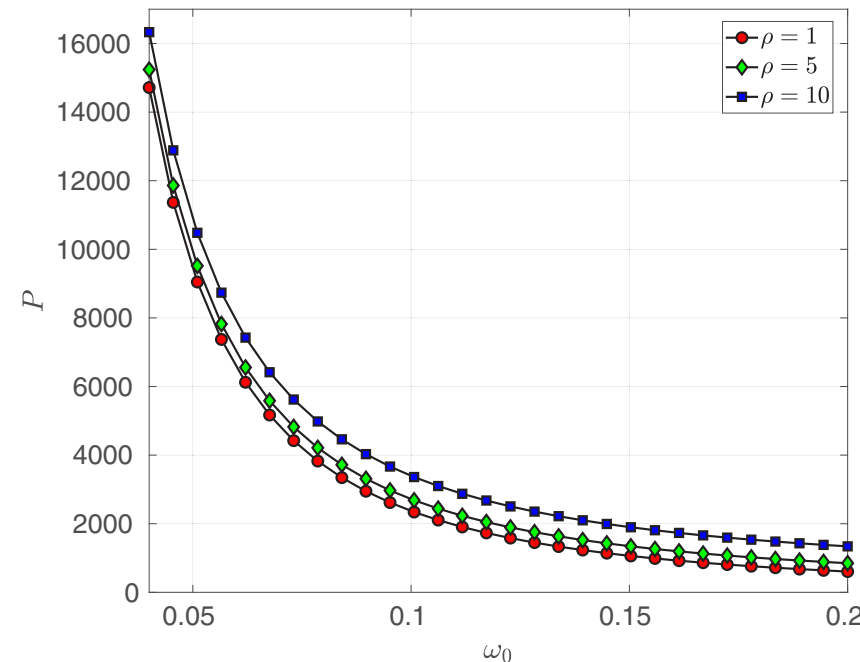

(b)

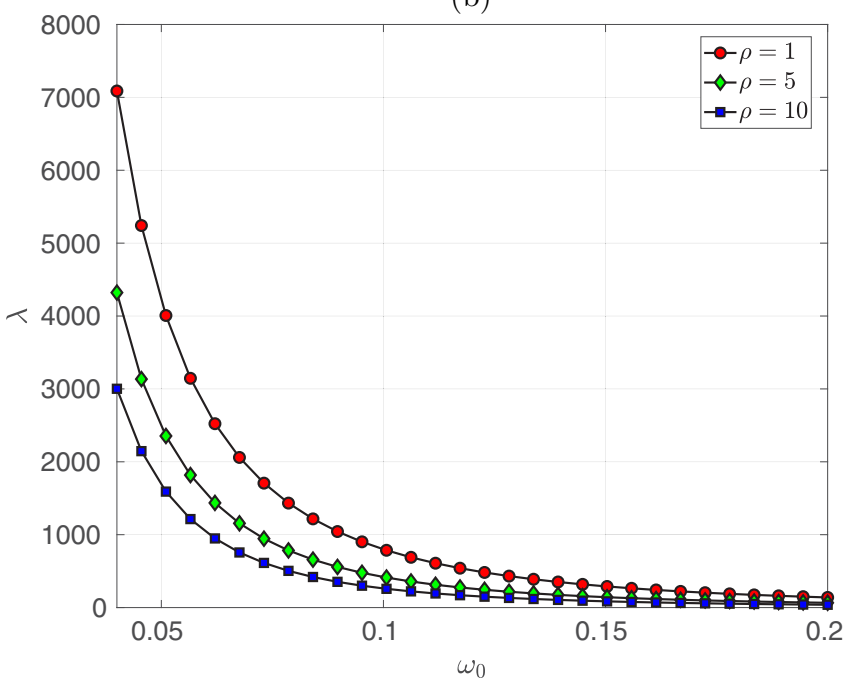

FIG. 4. (a) Soliton power and (b) propagation constant as a function of the beam width.

accuracy. Through comparison with a full numerical propagation of fundamental solitons with a relatively small waist (see Fig. 1), we found that this simplified model describes very well the trajectories of the solitons, even for relativelylow-power beams of about $P \sim 700$ or higher, with the added benefit of being able to draw conclusions about the dynamics from the analytical expressions in (11).

The quality of the approximation depends on how good the approximate force formula in (11) models (7), thus Fig. 3 shows a comparison between these two quantities for different values of (constant) boundary conditions $N_{b}$, along with the corresponding pointwise normalized error. It can be seen that the analytical approximation yields a good approximation throughout the interior of the circular cylinder.

The main soliton parameter in (11) is the power, however the validity of the approximation depends directly on the soliton waist. In order to relate these two crucial parameters, Fig. 4(a) presents the soliton power as a function of $\omega_{0}$ for small-waist solitons $\left(\omega_{0} \leqslant 0.2\right)$. Figure $4(b)$, on the other

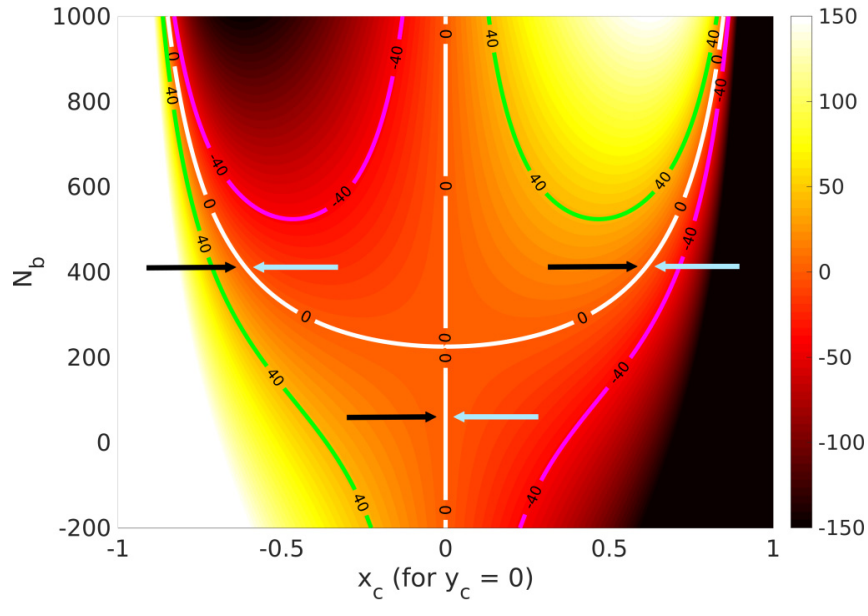

FIG. 5. Supercritical pitchfork bifurcation as the boundary condition $N_{b}$ (constant along the boundary) is increased. In this case, $P=800$ in a medium with $\rho=1$. The colormap represents the boundary force, and contour lines for force values of 0 and \pm 40 are also present. After the bifurcation, the center equilibrium point becomes unstable, while the two appearing equilibrium points are stable.

hand, presents the propagation constant $\lambda$ also as a function of $\omega_{0}$.

The examples in Figs. 1 and 3 show that when a nonzero boundary condition is present, the dynamical system that describes the soliton trajectory experiences a bifurcation of the center equilibrium point. In particular, a supercritical pitchfork bifurcation [32] is present when a constant boundary condition is applied, that is, as we increase the value of the boundary condition, the stable equilibrium point at the center degenerates into two equilibrium points, with the center point being unstable. In Fig. 5 we show the bifurcation diagram for this case, where instead of using $\mathbf{r}_{c}$ we took $y_{c}=0$ and plotted the diagram with respect to $x_{c}$ in order to illustrate the pitchfork behavior. The critical value of the boundary condition $N_{b}^{\text {crit }}$ at which the bifurcation occurs can be obtained as the value of $N_{b}$ for which the derivative of the force with respect to $N_{b}$ becomes zero at $x_{c}=0$ (with $y_{c}=0$ ) or equivalently by finding the value of $N_{b}$ that gives a zero eigenvalue on the linearization matrix of the dynamical system [32]. In Fig. 6 we present the behavior of $N_{b}^{\text {crit }}$ as a function of $\rho$, which shows that for small values of $\rho$, i.e., the highly nonlocal case, $N_{b}^{\text {crit }}$ tends to be high, while for $\rho>1$ the behavior is exponentially decaying. This result is consistent with the highly nonlocal case [19] for which the only equilibrium point is at the center of the cylinder, independently of the boundary condition. Additionally, Fig. 6 shows the behavior for different values of the soliton's power. Note that for constant $\rho$, the dependence of $N_{b}^{\text {crit }}$ as a function of $P$ is linear as a result from (11b) and (11b). For angle-dependent boundary conditions, we observed a similar behavior for which a new stable equilibrium region appears after a threshold value is exceeded [see Fig. 1(d) right plot].

Finally, we show that in the limiting case $\rho \rightarrow 0$ and for constant boundary conditions, we recover the same result as the one reported in [19]. Using the asymptotic forms of the modified Bessel functions for small arguments [33] and taking 


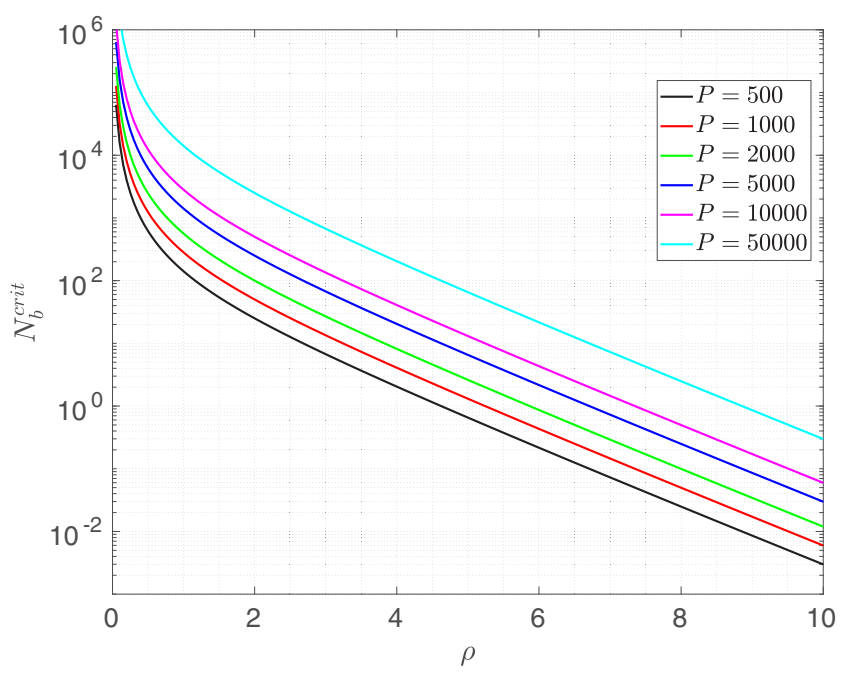

FIG. 6. Critical values of the boundary condition $N_{b}^{\text {crit }}$ as a function of the nonlocality parameter $\rho$ (semilogarithmic scale). For small $\rho$, the critical value of the boundary condition is large and in fact, as $\rho \rightarrow 0$, we have $N_{b}^{\text {crit }} \rightarrow \infty$, as expected from the purely local case, for which no bifurcation is present. From (11b) and (11b) we see that for a fixed $\rho$, the dependence on $P$ of $N_{b}^{\text {crit }}$ is linear.

the limit as $\rho$ goes to zero, we get

$$
\mathbf{F}_{2}\left(r_{c}\right)=-\frac{P}{2 \pi r_{c}} \sum_{n=1}^{\infty} r_{c}^{2 n} \hat{\mathbf{r}}=-\frac{P}{2 \pi} \frac{r_{c}}{1-r_{c}^{2}} \hat{\mathbf{r}} \quad \text { for } \rho \rightarrow 0
$$

which is exactly Eq. (8) from [19] (for constant boundary conditions $\mathbf{F}_{H} \rightarrow 0$ as $\rho \rightarrow 0$ ). In this particular case, the force is independent of the (constant) boundary condition, and only under an angle-dependent boundary condition does a force in the angular direction appear, which is given by

$$
\mathbf{F}_{H}\left(r_{c}, \theta_{c}\right)=\sum_{n=-\infty}^{\infty} i n r_{c}^{n-1} A_{n} e^{i n \theta_{c} \hat{\boldsymbol{\theta}}} \text { for } \rho \rightarrow 0 .
$$

This shows that the bifurcation of the equilibrium point does not occur in the highly nonlocal case, demonstrating that the introduction of the parameter $\rho$ in the model yields significantly different soliton dynamics.

\section{CONCLUSION}

In summary, we have obtained an analytical approximation for the force exerted on a soliton propagating in a finite nonlocal nonlinear media with a circular cylinder shape, with an arbitrary degree of nonlocality. This approximation can be used to predict the trajectories of small-waist soliton beams $\left(\omega_{0} \ll\right.$ 1) and is in good agreement with the numerical experiments presented. When the magnitude of the boundary condition exceeds a certain critical value, the original equilibrium point at the origin degenerates into an unstable equilibrium point and a stable equilibrium curve where the force is equal to zero; in the case of constant boundary conditions, this bifurcation is described by a supercritical pitchfork bifurcation. Recently, some analogies between optical soliton interactions and the dynamics of galactic cores in the scalar field dark-matter scenario have been reported [34]. We hope that a treatment similar to the one reported here can provide some insight into studies in this direction.

\section{ACKNOWLEDGMENT}

This work was supported by Consejo Nacional de Ciencia y Tecnología through Grant No. 243284.
[1] Y. S. Kivshar and G. Agrawal, Optical Solitons: From Fibers to Photonic Crystals (Academic press, New York, 2003).

[2] Z. Chen, M. Segev, and D. N. Christodoulides, Rep. Prog. Phys. 75, 086401 (2012).

[3] S. Skupin, O. Bang, D. Edmundson, and W. Krolikowski, Phys. Rev. E 73, 066603 (2006).

[4] Y. V. Kartashov, V. A. Vysloukh, and L. Torner, Opt. Express 15, 16216 (2007).

[5] M. Peccianti and G. Assanto, Opt. Express 15, 8021 (2007).

[6] X. Ma, Z. Yang, D. Lu, Q. Guo, and W. Hu, Phys. Rev. A 83, 033829 (2011).

[7] D. Buccoliero, A. S. Desyatnikov, W. Krolikowski, and Y. S. Kivshar, Phys. Rev. Lett. 98, 053901 (2007).

[8] S. Lopez-Aguayo, A. S. Desyatnikov, and Y. S. Kivshar, Opt. Express 14, 7903 (2006).

[9] S. Lopez-Aguayo and J. C. Gutiérrez-Vega, Opt. Express 15, 18326 (2007).

[10] D. Buccoliero, A. S. Desyatnikov, W. Krolikowski, and Y. S. Kivshar, J. Opt. A 11, 094014 (2009).

[11] S. Louis, T. R. Marchant, and N. F. Smyth, J. Phys. A: Math. Theor. 46, 055201 (2013).
[12] B. Alfassi, C. Rotschild, O. Manela, M. Segev, and D. N. Christodoulides, Opt. Lett. 32, 154 (2007).

[13] A. Alberucci, M. Peccianti, and G. Assanto, Opt. Lett. 32, 2795 (2007).

[14] C. Rotschild, B. Alfassi, O. Cohen, and M. Segev, Nat. Phys. 2, 769 (2006).

[15] A. B. Aceves, J. V. Moloney, and A. C. Newell, Phys. Rev. A 39, 1809 (1989).

[16] D. Brambila and A. A. Fratalocchi, Sci. Rep. 3, 2359 (2013).

[17] A. Fratalocchi and G. Assanto, Opt. Lett. 31, 1489 (2006).

[18] A. Fratalocchi, G. Assanto, K. A. Brzdakiewicz, and M. A. Karpierz, Opt. Lett. 31, 790 (2006).

[19] Q. Shou, Y. Liang, Q. Jiang, Y. Zheng, S. Lan, W. Hu, and Q. Guo, Opt. Lett. 34, 3523 (2009).

[20] A. Alberucci and G. Assanto, J. Opt. Soc. Am. B 24, 2314 (2007).

[21] A. I. Yakimenko, Y. A. Zaliznyak, and Y. Kivshar, Phys. Rev. E 71, 065603 (2005).

[22] N. Ghofraniha, C. Conti, G. Ruocco, and S. Trillo, Phys. Rev. Lett. 99, 043903 (2007).

[23] C. Conti, M. Peccianti, and G. Assanto, Phys. Rev. Lett. 91, 073901 (2003). 
[24] G. Assanto, M. Peccianti, and C. Conti, Opt. Photon. News 14, 44 (2003).

[25] D. G. Duffy, Green's Functions with Applications (CRC, Boca Raton, 2015).

[26] C. Pérez-Arancibia and M. Durán, J. Comput. Appl. Math. 235, 244 (2010).

[27] M.-C. Lai and W.-C. Wang, Numer. Methods Partial Differential Equations 18, 56 (2002).

[28] J. Weideman and B. Herbst, SIAM J. Numer. Anal. 23, 485 (1986).

[29] S. Zeng, M. Chen, T. Zhang, W. Hu, Q. Guo, and D. Lu, Phys. Rev. A 97, 013817 (2018).
[30] A. Alberucci, G. Assanto, D. Buccoliero, A. S. Desyatnikov, T. R. Marchant, and N. F. Smyth, Phys. Rev. A 79, 043816 (2009).

[31] A. Alberucci, C. P. Jisha, and G. Assanto, J. Opt. 18, 125501 (2016).

[32] L. Perko, Differential Equations and Dynamical Systems (Springer Science + Business Media, New York, 2013), Vol. 7.

[33] M. Abramowitz and I. A. Stegun, Handbook of Mathematical Functions: With Formulas, Graphs, and Mathematical Tables (Courier, Chelmsford, 1964), Vol. 55 .

[34] A. Navarrete, A. Paredes, J. R. Salgueiro, and H. Michinel, Phys. Rev. A 95, 013844 (2017). 